

\title{
Dielectric relaxations in a liquid crystal with helicoidal dipole ordering
}

Ludovic Benguigui

\section{To cite this version:}

Ludovic Benguigui. Dielectric relaxations in a liquid crystal with helicoidal dipole ordering. Journal de Physique, 1982, 43 (6), pp.915-920. 10.1051/jphys:01982004306091500 . jpa-00209469

\section{HAL Id: jpa-00209469 https://hal.science/jpa-00209469}

Submitted on 1 Jan 1982

HAL is a multi-disciplinary open access archive for the deposit and dissemination of scientific research documents, whether they are published or not. The documents may come from teaching and research institutions in France or abroad, or from public or private research centers.
L'archive ouverte pluridisciplinaire HAL, est destinée au dépôt et à la diffusion de documents scientifiques de niveau recherche, publiés ou non, émanant des établissements d'enseignement et de recherche français ou étrangers, des laboratoires publics ou privés. 


\title{
Dielectric relaxations in a liquid crystal with helicoidal dipole ordering
}

\author{
L. Benguigui \\ Solid State Institute, Technion-Israel Institute of Technology, Haifa, Israel
}

(Reçu le 11 décembre 1981, accepté le 23 février 1982)

\begin{abstract}
Résumé. - On a mesuré les relaxations diélectriques d'un cristal liquide ferroélectrique dans l'intervalle de fréquence $25 \mathrm{~Hz}-600 \mathrm{MHz}$, dans les deux cas où le champ électrique est parallèle ou perpendiculaire au plan des couches. On a trouvé deux relaxations dans la phase isotrope, quatre dans la phase $\mathrm{Sm} \mathrm{A}$ et sept dans la phase Sm C*. Il est possible de donner une interprétation satisfaisante des résultats en comparant avec les calculs théoriques si on admet que certains dipôles de la molécule participent à l'ordre hélicoïdal, tandis que d'autres n'y participent pas.
\end{abstract}

\begin{abstract}
We have measured the dielectric relaxations of a ferroelectric liquid crystal in a very large interval of frequency : from $25 \mathrm{~Hz}$ to $600 \mathrm{MHz}$ with the electric field parallel or perpendicular to the layer planes. We found two relaxations in the isotropic phase, four in the Sm A phase and seven in the $\mathrm{Sm} \mathrm{C}$ * phase. We give a satisfactory assignment of these relaxations using theoretical calculations, if we suppose that some molecular dipoles participate to the dipole ordering while others do not.
\end{abstract}

1. Introduction - The dielectric properties of the ferroelectric liquid crystals present a special interest in the field of liquid crystals since one can observe the influence of the helicoidal texture which is characteristic of these materials. The dielectric relaxations have been studied essentially on a theoretical basis first by Zeks and Blinc [1,2] and more recently by Martinot-Lagarde and Durand [3]. The essential difference between the two approaches, based on the same free energy expansion, is that the latter used the concept of normal modes. In their calculations, the relaxation frequencies are characteristic of the normal modes which are linear combinations of the physical variables.

The purpose of this paper is to determine experimentally the relaxation frequencies of the ferroelectric liquid crystal DOBAMBC (table I) : p-decycloxy -benzylidene-p-amino-2-methyl-butyl-ciannamate and to compare with the theoretical predictions.

At first glance, one may have the feeling that the results of Martinot-Lagarde and Durand are qualitatively very different from those given by Zeks and Blinc (compare figure 1 of reference [1] with figure 1 of reference [3]). In fact, one can easily observe that the results of Zeks and Blinc are valid only near $T_{c}$ and consequently they cannot be compared completely with those of reference [3], in which the calculation has been made on a large temperature interval [4].
Table I. - Formula and transition temperatures of $D O B A M B C$.

\section{DOBAMBC}

p-decycloxybenzylidene-p-amino-2-methylbutyl-cinnamate<smiles>C[C@@H](OC=CC=CC=[N+]=Cc1ccc(Oc2ccccc2)cc1)C(C)(C)C</smiles>

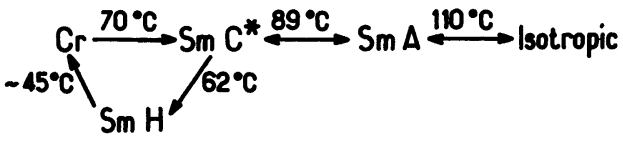

The concept of normal modes is new in the field of phase transition, it should be interesting : a) to compare the results of the two types of calculations; b) to compare them with the experimental results. Thus we have completed the calculation of Zeks and Blinc, which are based on the Landau-Khalatnikov equations. These equations give the differential equations of the various thermodynamic quantities, when deviations from the equilibrium appear. If we call $\psi(z)$, the tilt angle of the molecules and $P(z)$ the 
polarization in a plane, Zeks and Blinc have shown that for small deviations we have :

$$
\begin{aligned}
& \psi(z)=\psi_{q} \mathrm{e}^{i q z}+\psi_{0}+\psi_{2 q} \mathrm{e}^{2 i q z} \\
& P(z)=i\left(P_{q} \mathrm{e}^{i q z}+P_{0}+P_{2 q} \mathrm{e}^{2 i q z}\right)
\end{aligned}
$$

At equilibrium $\psi_{0}, P_{0}, \psi_{2 q}$ and $P_{2 q}$ are identically null and the two quantities $\psi(z)=\psi_{q} \exp i q z$ and $P(z)=i P_{q} \exp i q z$ have spatial variations giving the helicoidal structure. The dynamical equations of Landau [5] are :

$$
\begin{aligned}
& \frac{\partial \psi_{0}}{\partial t}=-\Gamma_{1} \frac{\partial F}{\partial \psi_{0}} \frac{\partial \psi_{2 q}}{\partial t}=-\Gamma_{1} \frac{\partial F}{\partial \psi_{2 q}} \\
& \frac{\partial P_{0}}{\partial t}=-\Gamma_{2} \frac{\partial F}{\partial P_{0}} \frac{\partial P_{2 q}}{\partial t}=-\Gamma_{2} \frac{\partial F}{\partial P_{2 q}} .
\end{aligned}
$$

In fact, in writing equations $(2 a)$ and $(2 b)$ Zeks and Blinc made several assumptions. First they supposed that the friction coefficient $\Gamma_{1}\left(\Gamma_{2}\right)$ is identical for $\psi_{0}\left(P_{0}\right)$ and $\psi_{2 q}\left(P_{2 q}\right)$. Secondly it is admitted that $\Gamma_{1}$ and $\Gamma_{2}$ are the same in the Sm A and the Sm C* phases. In fact, it is not certain that the dissipation process is not influenced by the structure. Third they supposed that there is no dissipation by interaction between the quantities $\psi_{0}, \psi_{2 q}, P_{0}$ and $P_{2 q}$ (no viscous coupling). One must emphasize that Martinot-Lagarde and Durand also made assumptions equivalent to these latter two. This may be a limitation when one wishes to make a comparison with the experimental results. Unfortunately we are not yet in a situation to make a quantitative comparison but only qualitative, and these assumptions seem to be justified at this time.

Using the free energy expansion as a function of the complex order parameters $\psi$ and $P$, as in reference [3], we calculated the dielectric relaxations, by means of the equations (2). We determined the relaxation frequencies and the associate amplitudes of the dielectric susceptibility, as functions of the temperature (more exactly as functions of the parameter $a$, coefficient of the term $|\psi|^{2}$ in the free energy expansion. As usual in the Landau theory of phase transitions, this parameter is linearly temperature dependent).

It is not necessary to give the results since they are qualitatively identical with those of Martinot-Lagarde and Durand [3]. Thus we refer the reader to their figure 6 giving the variation of the amplitudes versus the temperature. The true difference stands in the analytical values of the different quantities, but their temperature behaviours are the same. Since in this paper we cannot do more than qualitative analysis, with the help of our experimental results we are not able to distinguish between the two approaches : that using normal modes and that using the physical variables.
2. Experimental results. - We have measured the complex dielectric constant in a large frequency interval : between $25 \mathrm{~Hz}$ and $600 \mathrm{MHz}$ on a sample oriented by mean of a magnetic field in the three interesting phases : Isotropic, Smectic A and Smectic $\mathrm{C}^{*}$.

1. In the parallel configuration, the measuring electric field $E$ is parallel to the magnetic field. This means that $E$ is perpendicular to the layer. In the Sm A phase, we get a well oriented sample by cooling from the isotropic phase under a magnetic field of $22 \mathrm{kG}$. Once the sample is oriented in Sm A phase, the orientation persists in the $\mathrm{Sm} \mathrm{C*}$ phase.

2. In the perpendicular configuration, the measuring electric field is perpendicular to the orientating magnetic field : thus the electric field is parallel to the layer planes. We proceeded as above, by cooling from the isotropic phase, but in this case a magnetic field of $15 \mathrm{kG}$ is sufficient.

We used the following apparatuses to perform the measurements :

a) A bridge which was built in our laboratory for measurements between $20 \mathrm{~Hz}$ and $100000 \mathrm{~Hz}$.

b) A Boonton $Q$-meter for the range $50 \mathrm{kHz}$ $10 \mathrm{MHz}$.

c) A Hewlett-Packard impedancemeter for the measurements in the frequency interval : $0.5-100 \mathrm{MHz}$.

d) A network analyser associated with a HewlettPackard reflectometer in the range $50-600 \mathrm{MHz}$.

In the isotropic phase (above $110^{\circ} \mathrm{C}$ ) we observed two relaxations : one at a frequency of $50 \mathrm{MHz}$ and the second around $250 \mathrm{MHz}$. In figure 1 we show the variation of the two components of the complex

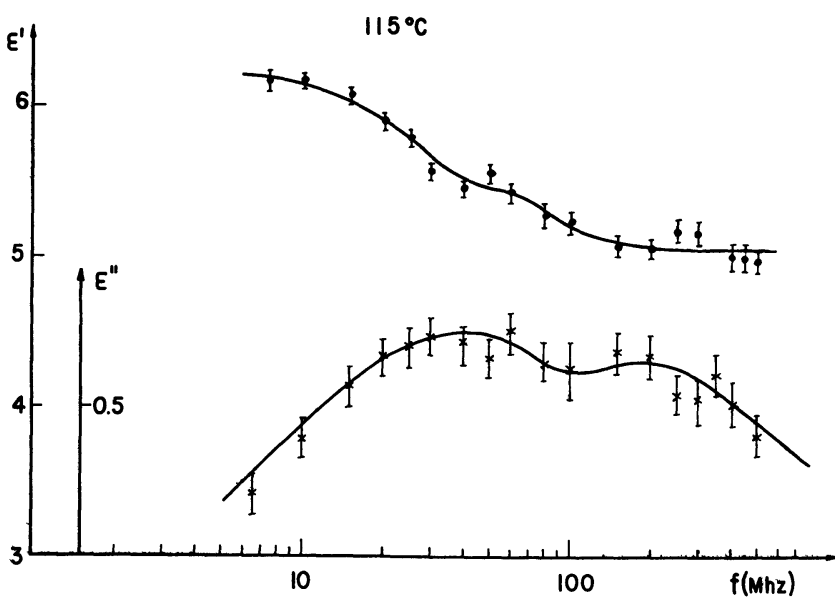

Fig. 1. - Variations of $\varepsilon^{\prime}$ and $\varepsilon^{\prime \prime}$ at $115^{\circ} \mathrm{C}$ (isotropic phase) as functions of the frequency.

dielectric constant $\varepsilon^{\prime}$ and $\varepsilon^{\prime \prime}$ with the frequency and in figure 2 we give the Cole-Cole diagram.

In the $\mathrm{Sm} \mathrm{A}$ phase (between $89^{\circ} \mathrm{C}$ and $110^{\circ} \mathrm{C}$ ) we get the following results (table II). In the parallel 
Table II. - Relaxations observed in the Sm A and Sm $\mathrm{C}^{*}$ phases, in the perpendicular $(\perp)$ and parallel $(/ /)$ configurations. $E$ is the measuring electric field and $H$ is the orientating magnetic field. In the $\mathrm{Sm} A$ phase the molecules are parallel to $H$.

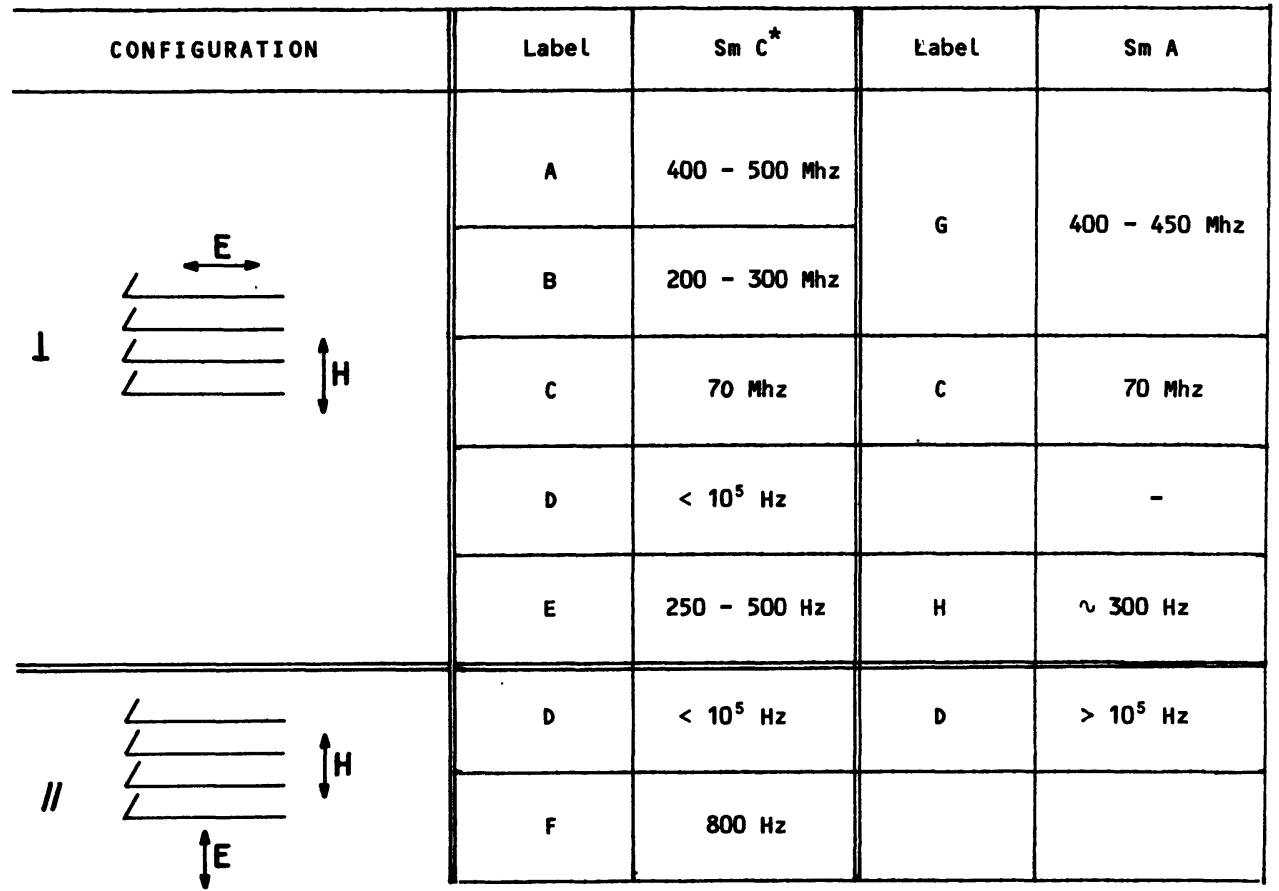

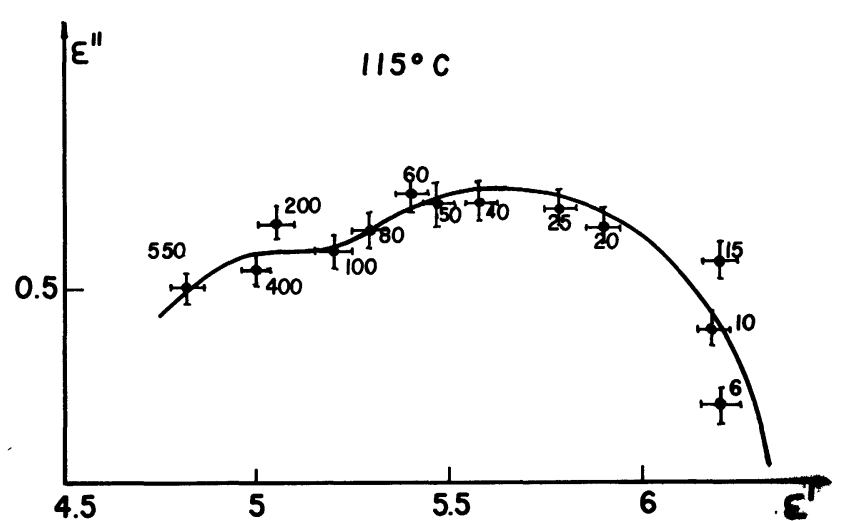

Fig. 2. - Cole-Cole diagram at $115^{\circ} \mathrm{C}$. One sees clearly the two relaxations as in figure 1 (Frequencies in $\mathrm{MHz}$ ).

configuration, we have only one relaxation with characteristic frequency above $10^{5} \mathrm{~Hz}$. The temperature dependence of the relaxation frequency is given by $f=f_{0} \mathrm{e}^{-w / k T}$ with $W \simeq 1.3 \mathrm{eV}$ (Fig. 3).

In the perpendicular configuration - always in the Sm A phase - we observed three relaxations. The lowest is observable only very near $T_{\mathrm{c}}\left(T-T_{\mathrm{c}} \lesssim 1{ }^{\circ} \mathrm{C}\right)$ at about $300 \mathrm{~Hz}$ (Fig. 4). The two others are located at much higher frequencies : at $70 \mathrm{MHz}$, and at $400 \mathrm{MHz}$. In figure 5, we show the Cole-Cole diagram at $95^{\circ} \mathrm{C}$ and one can see two relaxations : one around $70 \mathrm{MHz}$ and the second around $400 \mathrm{MHz}$. The lowest one has a relatively low amplitude. Because of the scatter of the experimental points, one can ask if

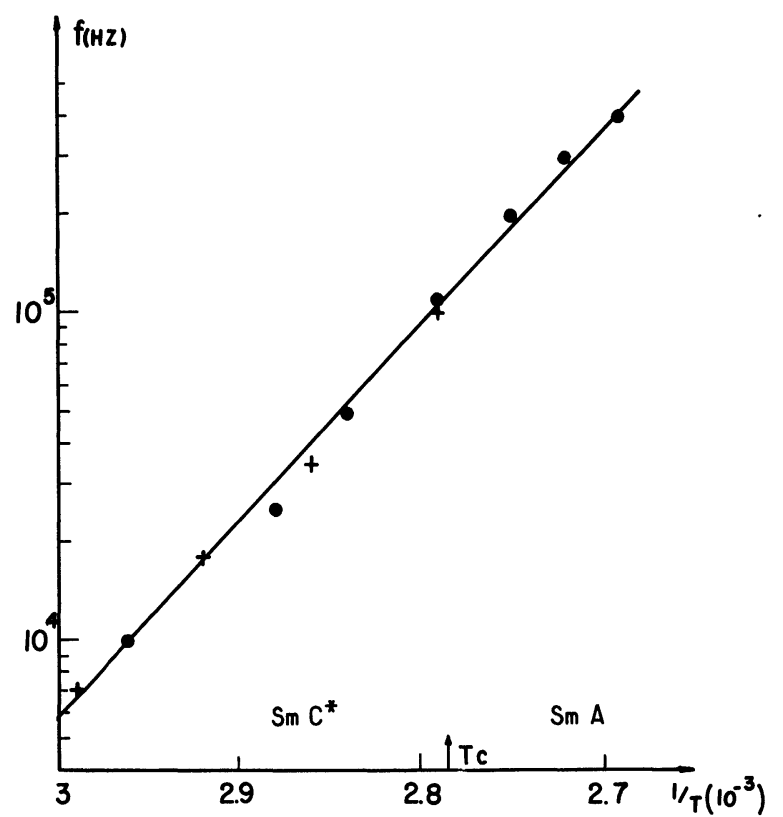

Fig. 3. - Dependence of the relaxation frequency with the inverse of the absolute temperature in the range $10^{4}-10^{6} \mathrm{~Hz}$. + perpendicular configuration, $\bullet$ parallel configuration. From the fact that the points corresponding to different phases and different configurations are all on a straight line, we conclude that we have the same relaxation mechanism.

there really are two relaxations or may be only one exists. However measurements over all the temperature range of the $\mathrm{Sm} \mathrm{A}$ phase showed that we have 


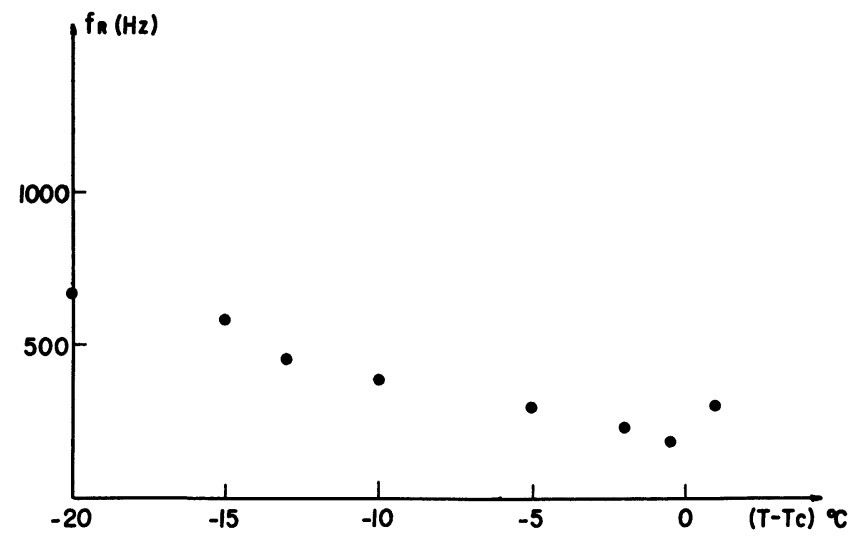

Fig. 4. - The lowest relaxations are observed in the range $600-250 \mathrm{~Hz}$ in the perpendicular configuration (Electric field parallel to the layer plane) in all the $\mathrm{Sm} \mathrm{C} \mathrm{C}^{*}$ phase and in the $\mathrm{Sm} \mathrm{A}, 1^{\circ} \mathrm{C}$ above the transition temperature.

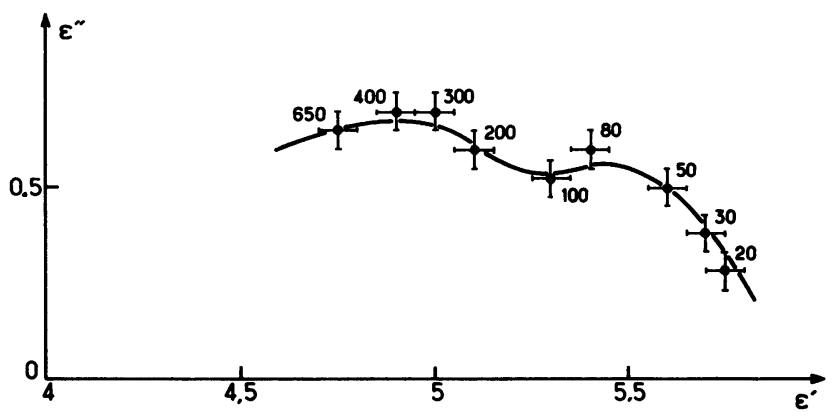

Fig. 5. - Cole-Cole diagram at $95^{\circ} \mathrm{C}$ in the Sm A phase. Frequencies in $\mathrm{MHz}$.

always the same disposition of the experimental points. Thus we concluded that there are two relaxations. In figure 6 we give the variations of the relaxation frequencies with the temperature, in the high frequency range.



Fig. 6. - Relaxation frequencies versus temperature in the high frequency range, in the isotropic, $\mathrm{Sm} \mathrm{A}$ and $\mathrm{Sm} \mathrm{C*}$ phases.
In the $\mathrm{Sm} \mathrm{C} \mathrm{C}^{*}$ (between 60 and $89^{\circ} \mathrm{C}$ ) we observed seven relaxations. In the parallel configuration, we got a relaxation at $800 \mathrm{~Hz}$, over all the $\mathrm{Sm} \mathrm{C} \mathrm{C}^{*}$ phase and a second below $10^{5} \mathrm{~Hz}$, with a frequency strongly dependent on the temperature. Plotting $\log f$ versus $1 / T$ (Fig. 3) we find that these points are aligned with those corresponding to relaxation observed in the $\mathrm{Sm}$ A phase above $10^{5} \mathrm{~Hz}$.

In the perpendicular configuration, we found four relaxations. The lowest has already been observed by several researchers [6] in the range $250-500 \mathrm{~Hz}$ and was attributed to the collective motion of the ordered dipoles (Fig. 4). The second one, below $10^{5} \mathrm{~Hz}$ has a frequency with exactly the same temperature dependence as that observed in the parallel configuration (Fig. 3), but with a lower amplitude. This relaxation was also observed by Blinov et al. [7]. Finally in the high frequency range we have three relaxations (Figs. 6 and 7).

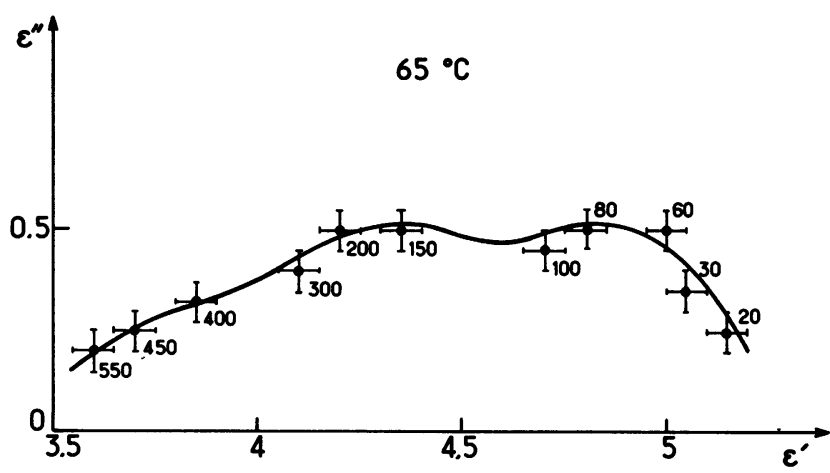

Fig. 7. - Cole-Cole diagram at $65^{\circ} \mathrm{C}$ in the $\mathrm{Sm} \mathrm{C} \mathrm{C}^{*}$ phase showing three relaxations. Frequencies in $\mathrm{MHz}$.

To resume, we have the following situation (see table II) : three groups of relaxations have been observed.

1. The high frequency relaxations above $50 \mathrm{MHz}$ : relaxations $\mathrm{A}, \mathrm{B}, \mathrm{C}$ and $\mathrm{G}$.

2. The relaxation $D$ with a frequency strongly dependent on $T$ (Fig. 3). Since in the two phases and in the two configurations (in the $\mathrm{Sm} \mathrm{C*}$ phase) we have the same temperature dependence, we think that we have one relaxation mechanism.

3. The low frequency relaxations around $500 \mathrm{~Hz}$ : relaxations $\mathrm{E}, \mathrm{F}$ and $\mathrm{H}$.

However we can propose another classification which is not based on the frequency domain. First, we have the relaxations which are affected by the phase transition $\mathrm{Sm} \mathrm{A}-\mathrm{Sm} \mathrm{C} \mathrm{C}^{*}$ at $89^{\circ} \mathrm{C}$ (relaxations A, $\mathrm{B}, \mathrm{C}$ and $\mathrm{E}, \mathrm{H}, \mathrm{F})$. Then the relaxations unaffected by the phase transition. Their variations with the temperature do not show any anomalies at the transition temperature : relaxations $\mathrm{C}$ and $\mathrm{D}$.

3. Discussion. - The observation of two relaxations in the isotropic phase (in our frequency range) 
is completely unusual in liquid crystal. And we think that this can be related to the second classification of the relaxations, that we proposed above. Thus we shall interpret all our experimental results using the following basic assumption. The molecule of DOBAMBC has several dipoles (see the molecular formula in table I) and we assume that they can be divided into two groups : one has the habitual dielectric behaviour observed in the liquid crystal phases and does not participate in the dipole ordering parallely to the layer planes. The second group is ordered in the $\mathrm{Sm} \mathrm{C}^{*}$ and these dipoles are responsible for all the particular properties of this ferroelectric liquid crystal. It is likely that these ordering dipoles are located on the chain with the asymmetric carbon.

It is now well established [8] that in the liquid crystals phases (at least in the nematic, $\mathrm{Sm} \mathrm{A}$ and $\mathrm{Sm} \mathrm{C*}$ phases) we can observe two types of relaxations associated with the molecular rotations : one (flipflop) corresponds to the rotation around a short axis and the other to the rotation around the long axis. Their characteristic frequencies are very different as well as their temperature dependence. In the first case, the relaxation frequency is strongly temperature dependent, with an activation energy of the order of $1 \mathrm{eV}$. And the frequency range is well below the characteristic frequency of the relaxation in the isotropic phase. On the contrary in the second case (rotation around the long axis) the relaxation frequency has a slow temperature dependence and its value is slightly above the isotropic relaxation frequency.

It is easy to observe that the relaxations $\mathrm{D}$ and $\mathrm{C}$, associated with the relaxation at $50 \mathrm{MHz}$ in the isotropic phase have exactly the behaviour we described in the preceding paragraph. The relaxation $\mathrm{D}$ with its activation energy of $1.36 \mathrm{eV}$ is associated with the rotation around a short axis. We note that it is observed in the Sm A phase only if the electric field is perpendicular to the layer, i.e., parallel to the molecules. However, in the Sm C* phase, it is observed in the two configurations since the electric field is never perpendicular to the molecules (in which case we do not observe this relaxation) due to the tilt angle. This is in agreement with the fact that in the perpendicular configuration, the amplitude increases when the temperature decreases or when the tilt angle increases. This effect has been already observed by Buka and Bata [9] in their measurement in the $\mathrm{Sm} \mathrm{C}$ phase. The relaxation frequency $\mathrm{C}$ is slightly above the relaxation frequency in the isotropic phase, thus we assign it to the rotation around the long axis.

Now we deal with the second group of dipoles. Following our assumption, they are responsible for the relaxation in the isotropic phase at $250 \mathrm{MHz}$ and for all the other relaxations. We shall try to compare them with the theoretical results of Martinot-Lagarde and Durand [3].
First, we begin with the $\mathrm{Sm} \mathrm{C} \mathrm{C}^{*}$ phase. We see on the figure 6 of reference [3] that the theory predicts four relaxations but we have observed only three. The "Goldstone mode" (mode +1$)$ is without doubt the relaxation $\mathrm{E}$ and it is easy to verify [10] that the frequency is approximately proportional to $q^{2}$, where $q$ is the inverse of the helix pitch. Comparing figure 6 of reference [3] and our figure 6 it is tempting to say that the modes +2 and -2 are the relaxations $A$ and $B$, and that the mode -1 is not observed because of too low an amplitude. But this assignment is not compatible with the calculated amplitudes : the strongest mode is the mode +1 but the lowest is the mode +2 . If one mode is not observed it should be the mode +2 . The mode -1 has the particularity to vary strongly with the temperature, from very low values $(500 \mathrm{~Hz}$ at $T_{\mathrm{c}}$ ) up to several hundreds of megahertz far from $T_{\mathrm{c}}$. Apparently this mode is not observed, in spite of a large amplitude.

We have the same problem in the Sm A phase. We may identify the relaxations $G$ with the mode 2 (high frequency range) and the relaxation $\mathrm{H}$ with the mode 1 . However, we have observed the relaxation $\mathrm{H}$ only near $T_{\mathrm{c}}$ and this is in contradiction with the calculated amplitude. It is predicted to be very strong and we should be able to detect it at all temperatures.

Following Durand [11] we can resolve the difficulties by admitting that the slopes of the modes -1 and 1 are very high and consequently they have an observable temperature dependence only very near $T_{\mathrm{c}}$. Because of this steep increase, it is difficult to observe the temperature dependence of these relaxations. Consequently in our measurements, because the lack of very precise temperature determination $\left( \pm 0.2^{\circ} \mathrm{C}\right)$ and the presence of an estimated thermal gradient of $0.2^{\circ} \mathrm{C}$, we were not able to observe this rapid increase of the modes -1 and 1 . In fact, we observe these modes only at their saturation. We drew schematically (Fig. 8) the temperature variation of the frequency rela-

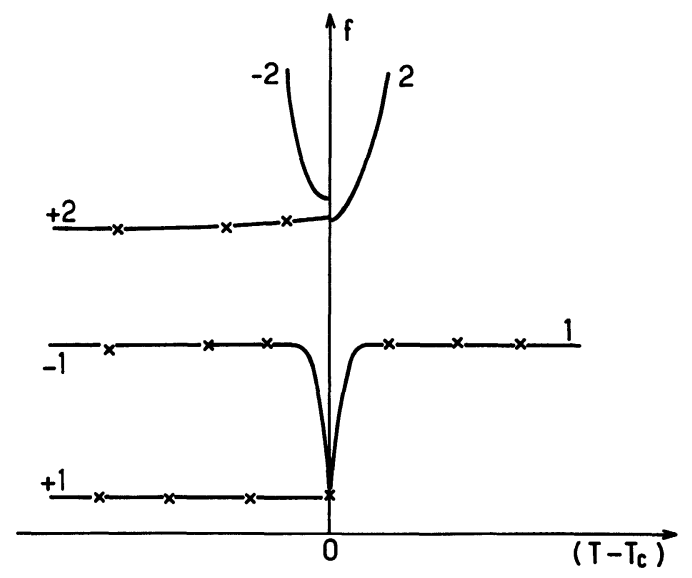

Fig. 8. - Schematic variations of the relaxation frequencies versus the temperature. The crosses indicate the measurements. Due to the rapid variation of the modes -1 and 1 near $T_{\mathrm{c}}$, we measured only the saturation values giving the impression that there is no anomaly at $T_{\mathrm{c}}$. In fact we observed a change in the slope. 
xations and we marked by crosses the measurements. Following this interpretation we have the following assignment :

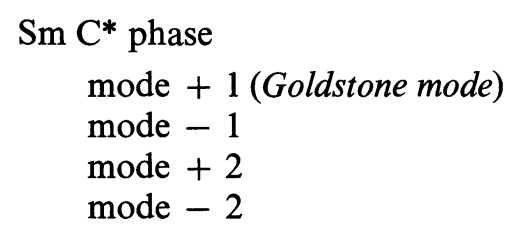

Relaxation E
Relaxation B
Relaxation A
Unobserved

Sm A phase

mode 1

mode 2

\section{$\{$ Relaxation $\mathrm{H}$ \\ Relaxation G \\ Unobserved}

The modes -2 and +2 are not observed because of their low amplitude and may be also because of their very high relaxation frequencies. Furthermore, in the $\mathrm{Sm} \mathrm{C}^{*}$, the amplitudes observed are in qualitative agreement with the predictions. In particular the relaxation A has a relatively low amplitude.

There is one relaxation which we have not yet discussed. It is the relaxation $\mathrm{F}$, observed in the $\mathrm{Sm} \mathrm{C}^{*}$, phase in the parallel configuration. We propose [10] to attribute it to motion of the ordered dipoles out of the layer planes when the electric field is perpendicular to them.

Finally we want to emphasize that we made a qualitative analysis only. The theoretical calculation does not take into account the temperature dependence of the different parameters, except the coefficient $a$. Thus the reader must not be confused by our assignment of modes which are indicated (i.e. mode +2 , saturation of modes -1 and 1) as temperature independent on the theoretical curves with relaxations (A, B and G) which are in fact temperature dependent.

4. Conclusion - We have observed a large number of relaxations in the isotropic, $\mathrm{Sm} \mathrm{A}$ and $\mathrm{Sm} \mathrm{C} \mathrm{C}^{*}$ phases of the ferroelectric liquid crystal DOBAMBC, in the two possible configurations : electric field perpendicular to the layers or electric field parallel to them.

Our interpretation is based on the asumption that the molecular dipoles can be divided into two groups : in the first, the dipoles do not participate in the dipole ordering and consequently behave as in other liquid crystals. On the other hand, there are other dipoles which are ordered in the $\mathrm{Sm} \mathrm{C} \mathrm{C}^{*}$ phase. We have compared their behaviour with the theoretical predictions. We succeeded in the identification of the various relaxations only if we suppose very steep variations near $T_{\mathrm{c}}$ of two modes (one below and the other above $T_{\mathrm{c}}$ ). However, we have not observed all the modes predicted by the theory. These modes have relatively low frequency and high amplitude only very near $T_{\mathrm{c}}$. Thus it should be very interesting to perform measurements in the immediate vicinity of $T_{c}$ in order to detect all the modes (four below and two above $T_{\mathrm{c}}$ ) and their temperature dependence.

Acknowledgments. - I thank MM. G. Durand and $\mathrm{Ph}$. Martinot-Lagarde from the «Laboratoire de Physique des Solides, Orsay " for kindly furnishing the material and for fruitful discussions.

\section{References}

[1] Blinc, R. and Zeks, B., Phys. Rev. A 18 (1978) 740.

[2] Zek's, B., Levstik, A. and Blinc, R., J. Physique Colloq. 40 (1979) C 3-409.

[3] Martinot-Lagarde, Ph. and Durand, G., J. Physique 42 (1981) 269.

[4] Because we do not know the values of the different constants used in the calculations, it is difficult to give estimations of what we call « large interval » or " near $T_{\mathrm{c}}$ ".

[5] Landau, L. et Lifchitz, E., Physique Statistique (Editions Mir, Moscou) 1967, ch. 12, § 122.

[6] a) Hoffmann, J., KuCZyUSKI, W. and MaleCKI, J., Mol. Cryst. Liq. Cryst. 44 (1978) 287.

b) OstrowskiI, B. I., Rabinovitch, A. Z., Sonin, A. S. and Strukov, B. A., Sov. Phys. JETP 47 (1978) 912. c) Levstik, A., Zeks, B., Levstik, I., Blinc, R. and FIliPIC, C., J. Physique Colloq. 40 (1979) C 3303.

[7] Blinow, L. M., Beresnev, L. A., Shtykov, N. M. and Elashvili, Z. M., J. Physique Colloq. 40 (1979) C 3-269.

[8] De Jeu, W. H., Liquid Crystals, Solid State Phys. Supp. $N^{\circ}$ 14, L. Liebert Ed. (Academic Press, N.Y.) 1978 , p. 109.

[9] Bata, L. and BuKa, A., Advances in Liquid Crystal Research and Applications, L. Bata Ed. (Pergamon Press-Oxford) 1980 , p. 251.

[10] Benguigui, L., Submitted for publication.

[11] Durand, G., Private communication. 